Supporting Information

\title{
Reversely Orthogonal Actuation of Janus-faced Film Based on Asymmetric Polymer Brushes Modification
}

Yangyang Xiang ${ }^{a, b}$, Bianhong Li ${ }^{e}$, Yunlei Zhang ${ }^{c}$, Shuanhong $\mathrm{Ma}^{b}$, Bin Li ${ }^{2}$, \#, Hanjun Gao ${ }^{d}$, Bo $\mathrm{Yu}^{b *}$, Jian $\mathrm{Li}^{a *}$, Feng Zhou ${ }^{b}$

a Gansu International Scientific and Technological Cooperation Base of Water-retention Chemical Functional Materials, Northwest Normal University, Lanzhou 730070, China

${ }^{b}$ State Key Laboratory of Solid Lubrication, Lanzhou Institute of Chemical Physics, Chinese Academy of Sciences, Lanzhou 730000, China

${ }^{c}$ University of Chinese Academy of Sciences, Beijing 100039, China

${ }^{d}$ School of Mechanical Engineering and Automation Beihang University, Beijing 100191, China

e School of Mechatronical Engineering Beijing Institute of Technology, Beijing 100081, China

\#Physik Department, Technische Universität München, James-Franck-Straße 1, 85748 Garching, Germany.

*Email:yubo@licp.cas.cn 
*Email: jianli83@126.com

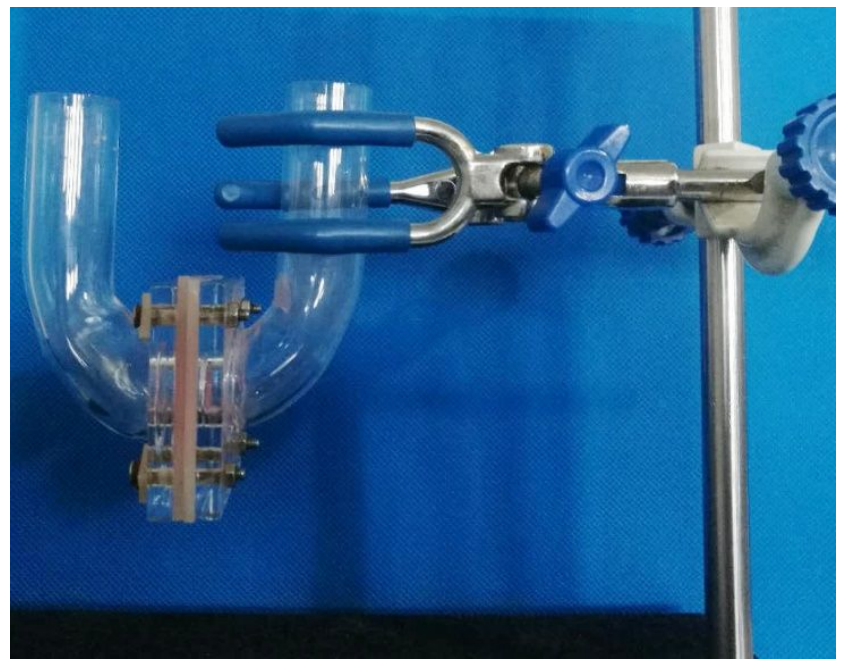

Figure S1. Image of the experimental setup for asymmetric polymer brushes modification.

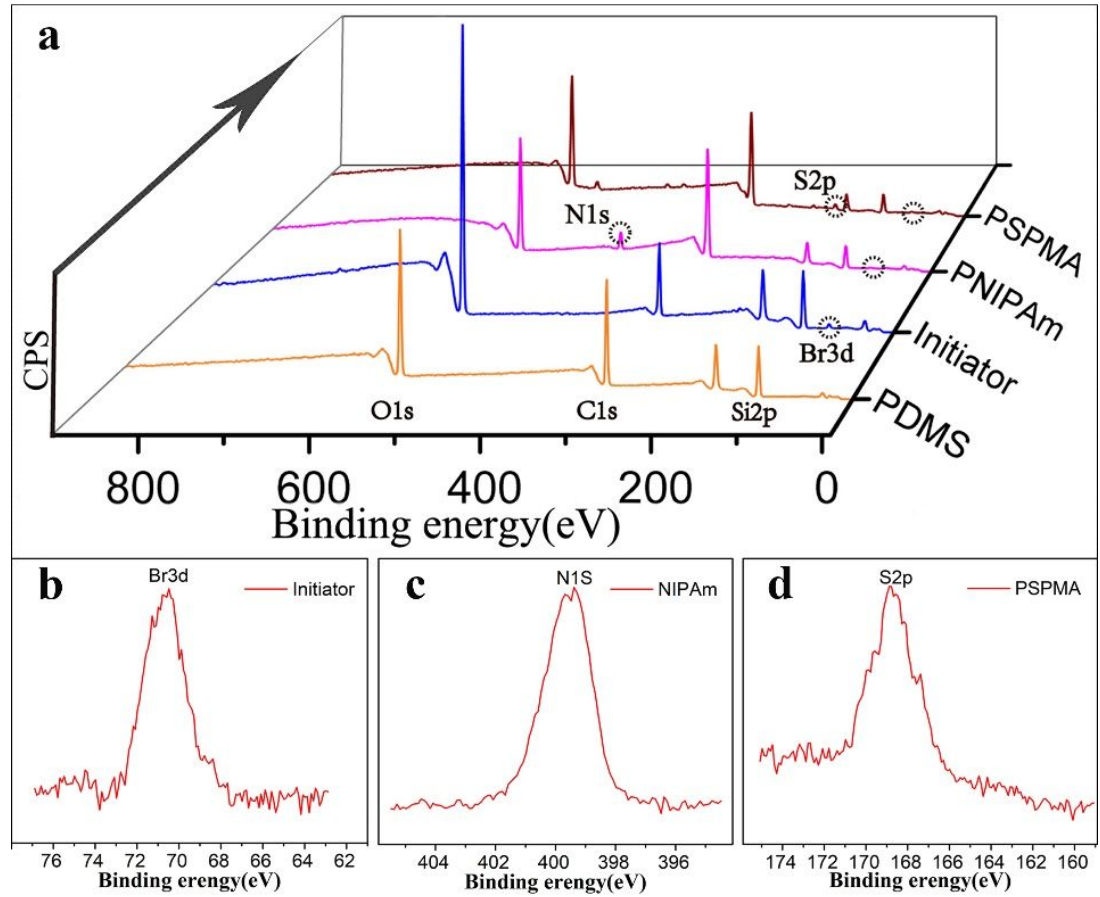

Figure S2. The XPS survey of chemical composition for the different samples. (a)

Surface composition of asymmetric modified films (The order of preparation is 
indicated by the direction of the black arrow in the figure). (b) Characteristic element of Br3d after modified initiator upon film. (c) Characteristic element of N1s after modified PNIPAAm brush upon film and (d) Characteristic element of S2p after modified PSPMA brush upon film.

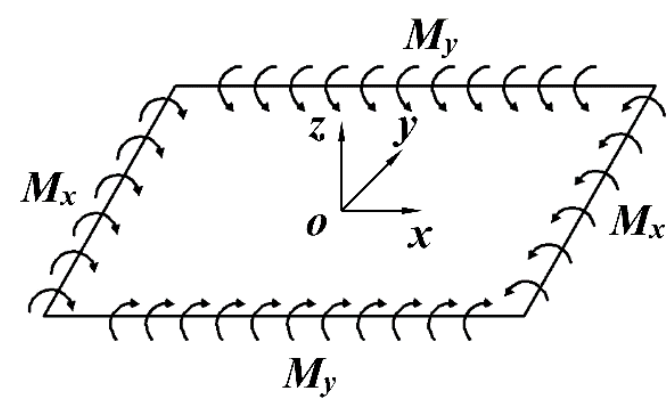

Figure. S3. Boundary conditions of the film subjected to uniform bending moment.
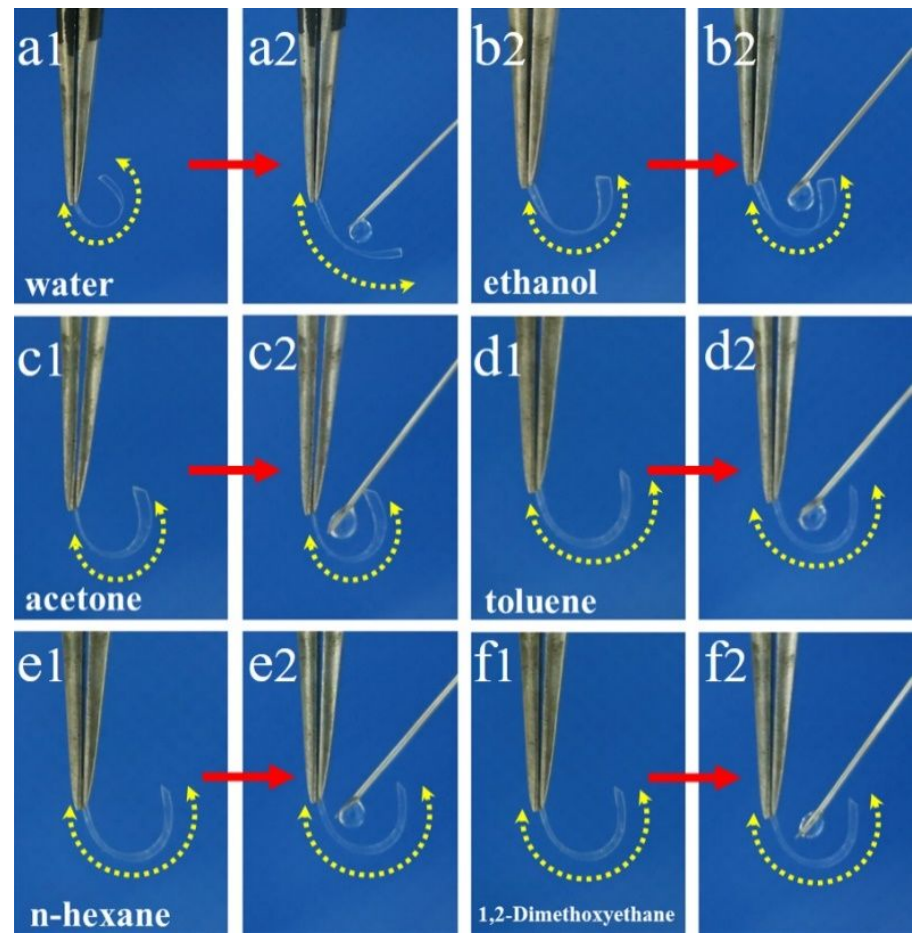

Figure S4. Intelligently identify different solvents of PDMS film asymmetrically modified by polymer brushes with non-touch mode. (a1, 2) A water droplet; (b1, 2) 
An ethanol droplet; $(\mathrm{c} 1,2)$ An acetone droplet; $(\mathrm{d} 1,2)$ A toluene droplet; $(\mathrm{e} 1,2)$ A n-hexane droplet; (f1, 2) A 1, 2-Dimethoxyethane droplet.

$a_{1}$
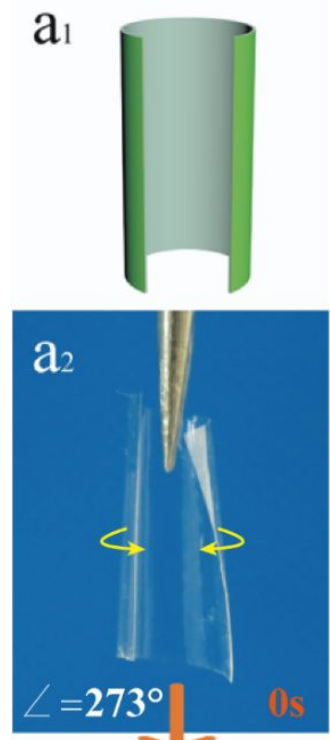

$\mathrm{e}_{1}$
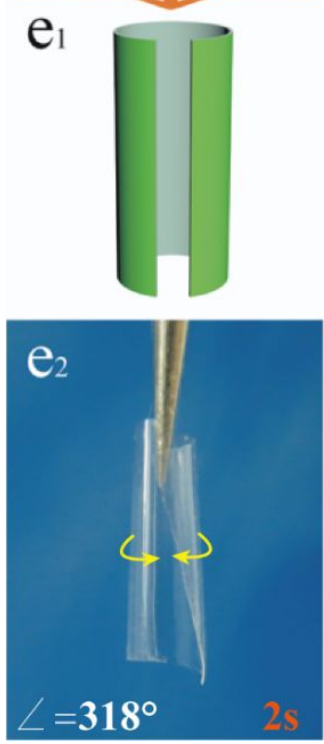

$b_{1}$
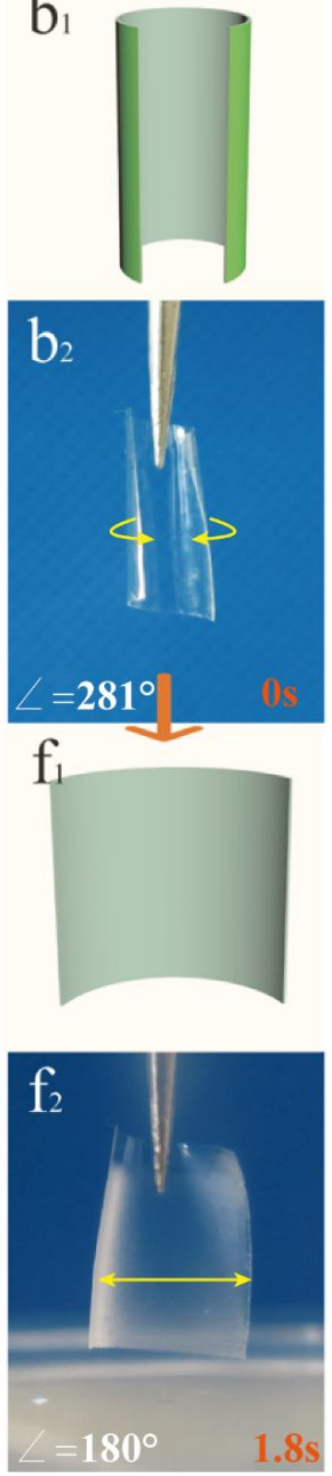

$\mathrm{c}_{1}$
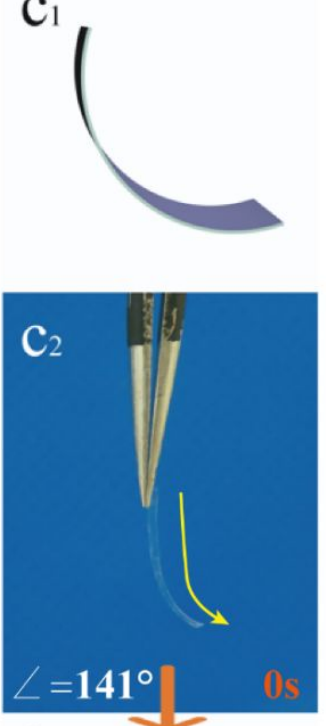

$\mathrm{g}_{1}$
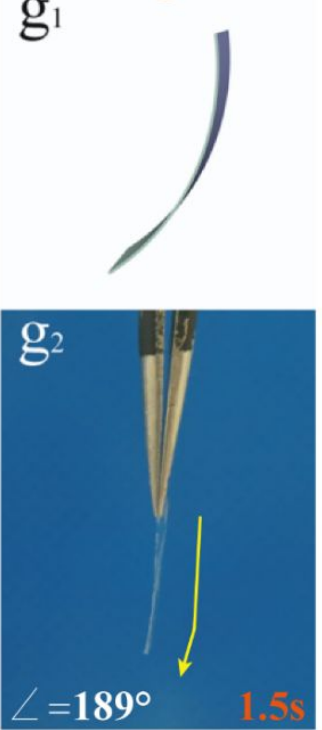
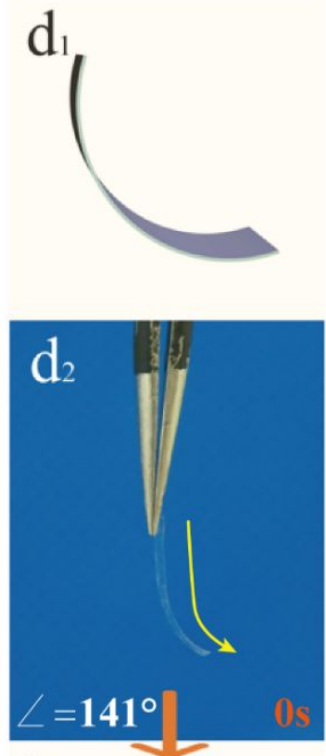

$\mathrm{h}_{1}$
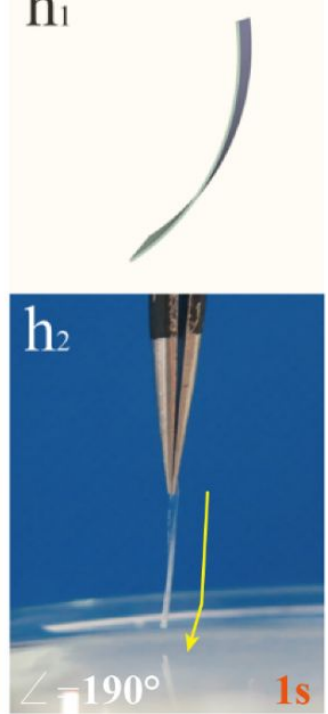

Figure S5. PDMS modified by single-side polymer brush. (a, b, e, f) Film modified by PNIPAAm brush; (c, d, g, h) Film modified by PSPMA brush. $\left(\mathrm{a}_{1} \mathrm{~h}_{1}\right)$ Photos of bending direction according to the actual experimental phenomena; (e, g) Temperature is about $25^{\circ} \mathrm{C}$ and $(\mathrm{f}, \mathrm{h})$ Temperature is about $40{ }^{\circ} \mathrm{C}$. 